\title{
T2K oscillation results
}

\author{
Lorenzo Magaletti ${ }^{* \dagger}$ \\ INFN Sezione di Bari \\ E-mail: lorenzo.magaletti@ba.infn.it
}

The T2K (Tokai-to-Kamioka) experiment is a second generation long baseline neutrino oscillation experiment that probes physics beyond the Standard Model. An off-axis neutrino beam with a peak energy of $\sim 0.6 \mathrm{GeV}$ is produced at the J-PARC accelerator facility, with the flavour content dominated by either muon neutrinos or muon anti-neutrinos, depending on the choice of the polarity of the magnetic focusing horns. The neutrino beam is detected first in the near detector ND280, where the flavour composition of the incoming neutrino flux is not expected to be affected by oscillation, and then travels $295 \mathrm{~km}$ to the far detector Super-Kamiokande, where oscillation significantly affects the flavour composition. We report the results of a joint analysis of neutrino and antineutrino oscillations at T2K with the $v_{\mu}\left(\bar{v}_{\mu}\right)$ disappearance and $v_{\mu}\left(\bar{v}_{\mu}\right) \rightarrow$ $v_{e}\left(\bar{v}_{e}\right)$ appearance channels, obtained by collecting a total statistic of $7.57 \times 10^{20}$ protons-ontarget in $v$-mode and $7.53 \times 10^{20}$ in $\bar{v}$-mode. The results in the disappearance channel show that our data continue to prefer maximal $\theta_{23}$ mixing $\left(\sin ^{2} \theta_{23}=0.532_{-0.068}^{+0.046}\right.$, in case of normal mass hierarchy) and no CPT violation, while in the appearance channel, we observed a large $v_{e}$ appearance and a low $\bar{v}_{e}$ appearance with respect to the expectations. These results favour a $\delta_{C P} \sim-\pi / 2$, with a $90 \%$ confidence interval of $[-3.13,-0.39]$ in normal mass hierarchy and $[-2.09,-0.74]$ in inverted mass hierarchy. We comment briefly on the future prospects for T2K, including a proposal for extended running to accumulate $20 \times 10^{21}$ protons-on-target, nearly three times the currently approved amount by 2026, to gain substantial sensitivity to CP violating effects in $v_{\mu}\left(\bar{v}_{\mu}\right) \rightarrow v_{e}\left(\bar{v}_{e}\right)$ oscillations if parameters are favorable.

Neutrino Oscillation Workshop

4 - 11 September, 2016

Otranto (Lecce, Italy)

* Speaker.

${ }^{\dagger}$ on behalf of the $\mathrm{T} 2 \mathrm{~K}$ collaboration. 


\section{The T2K experiment}

The Tokai-to-Kamioka (T2K) experiment [1] uses a $30 \mathrm{GeV}$ proton beam from the J-PARC accelerator facility in Tokai (Ibaraki prefecture, Japan), to produce a high purity muon neutrino beam, which is detected first at $280 \mathrm{~m}$ from the neutrino production point in the near detector complex (composed by ND280 and INGRID detectors), where the flavour composition of the incoming neutrino flux is not expected to be affected by oscillation, and then travels $295 \mathrm{~km}$ to the far detector Super-Kamiokande [2] (Gifu prefecture, Japan), where oscillation significantly affects the flavour composition. The near detector ND280 and the far detector Super-Kamiokande (SK) are placed $2.5^{\circ}$ off-axis with respect to the neutrino beam centre, resulting in a quasi-monochromatic neutrino energy spectrum that is sharply peaked around $0.6 \mathrm{GeV}$ in order to enhance the effect of neutrino oscillations at $295 \mathrm{~km}$. The neutrino flux is obtained by hitting accelerated protons on a graphite target. A set of three pulsed electromagnets ("horn") focuses either positive pions into a heliumfilled decay region to produce a beam primarily composed of $v_{\mu}$ ( $\nu$-mode), or negative pions to produce a $\bar{v}_{\mu}(\bar{v}$-mode) enhanced beam. T2K can investigate two neutrino oscillation channels and two anti-neutrino oscillation channels: $v_{\mu}\left(\bar{v}_{\mu}\right)$ disappearance and $v_{e}\left(\bar{v}_{e}\right)$ appearance. In the disappearance channel, the oscillation probability $P\left(v_{\mu} \rightarrow v_{\mu}\right)$, is sensitive to $2 \theta_{23}$ and $\Delta m_{32}^{2}$. In the appearance channel, the oscillation probability $P\left(v_{\mu} \rightarrow v_{e}\right)$ is sensitive to $\theta_{13}$ and the octant of $\theta_{23}$ in the leading term, and is sensitive to $\sin \delta_{C P}$ and matter effect in the sub-leading terms. At $\mathrm{T} 2 \mathrm{~K}$, the CP-violating phase $\delta_{C P}$ has an effect up to $\sim 30 \%$, while the matter effect has a lower impact $(\sim 10 \%)$. This will result in asymmetries in the probabilities for the $\mathrm{CP}$-conjugate channels $v_{\mu} \rightarrow v_{e}$ and $\bar{v}_{\mu} \rightarrow \bar{v}_{e}$ if $\sin \delta_{C P} \neq 0$ or $\pm \pi$, with negative (positive) values of $\sin \delta_{C P}$ enhancing (suppressing) $v_{\mu} \rightarrow v_{e}$ oscillations and suppressing (enhancing) $\bar{v}_{\mu} \rightarrow \bar{v}_{e}$ oscillations.

\section{Predicted neutrino flux}

The neutrino flux from the $\mathrm{T} 2 \mathrm{~K}$ beam line is predicted from a data-driven simulation based on Geant3-based Monte Carlo, incorporating data from the NA61/SHINE experiment [3, 4], which has provided critical measurements of the hadron production cross sections using a thin carbon and a T2K replica target [5]. In addition, beam monitor data and the beam direction, profile and stability, measured by the on-axis near detector INGRID [6], are incorporated into the flux prediction and its systematic errors, which are reduced at the level of $\sim 10 \%$ [7]. The predicted neutrino fluxes in both $v$ and $\bar{v}$ modes at SK are shown in Fig. 1.

\section{Near detector analysis}

The off-axis near detector ND280 consists of a number of sub-detectors installed inside the refurbished UA1/NOMAD magnet, which provides a $0.2 \mathrm{~T}$ field to identify the charge of the particle passing through ND280. $v_{\mu}$ and $\bar{v}_{\mu}$ Charged Current (CC) events are selected in the tracker region of ND280, which consists of three time projection chambers (TPC1, 2, 3) [8], interleaved with two fine-grained detectors (FGD1, 2) [9]. The upstream FGD1 detector consists of 15 scintillator modules, while the downstream FGD2 contains seven scintillator modules alternating with six water modules. The observed energy loss in the tracker is used for particle identification which, 

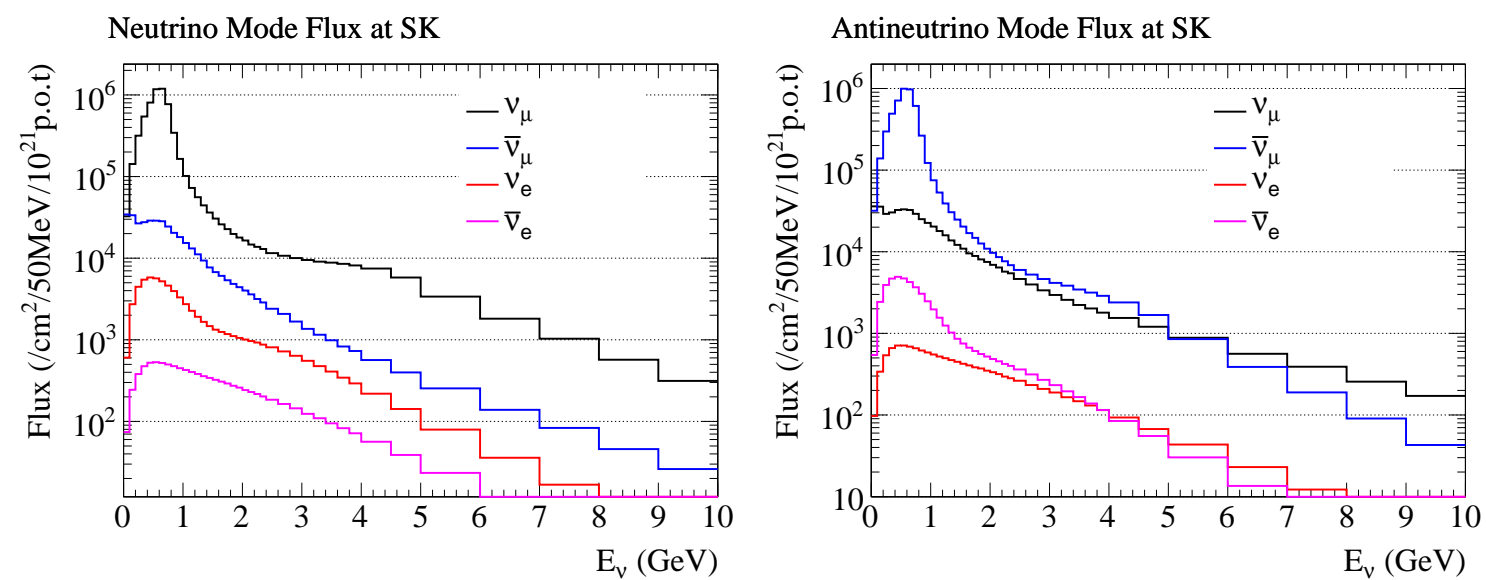

Figure 1: Predicted flux of neutrinos and antineutrinos by species at the SK detector in the absence of oscillation effects for $v$-mode (left) and $\bar{v}$-mode (right).

when combined with particle charge information, allows a precise separation and measurement of the $\bar{v}_{\mu}$ (right-sign) and $v_{\mu}$ (wrong-sign) in the $\bar{v}$-mode. In the $v$-mode case, muon neutrino induced $\mathrm{CC}$ interactions are selected in the FGDs Fiducial Volume (FV). Than the $v_{\mu} \mathrm{CC}$ candidates are divided into three subsamples, according to the number of identified pions in the event: CC- $0 \pi$, $\mathrm{CC}-1 \pi^{+}$and CC-Other, which are dominated by "quasi-elastic" (CCQE), CC resonant pion production, and deep inelastic scattering interactions, respectively [10]. For the $\bar{v}$-mode case, both $\bar{v}_{\mu}$ and $v_{\mu} \mathrm{CC}$ interactions are selected and then divided into two subsamples, defined by the number of reconstructed tracks crossing the TPCs: $\bar{v}_{\mu}\left(v_{\mu}\right)$ CC-1-track, dominated by CCQE interactions and $\bar{v}_{\mu}\left(v_{\mu}\right)$ CC-N-tracks $(\mathrm{N}>1)$, a mixture of resonant production and deep inelastic scattering [11]. The major update in the near detector analysis is the use of interactions in FGD2. By including both FGD1 and FGD2 samples, resulting in a total of six samples in $v$-mode and eight samples in $\bar{v}$-mode, the interaction properties on water can be effectively isolated, reducing the uncertainties related to extrapolating across differing nuclear targets in the near and far detectors. The ND280 analysis performs a simultaneous fit of the fourteen samples in muon momentum and polar angle distributions, in order to constrain parameters representing the systematic uncertainties in the neutrino flux and interaction models. The muon momentum distributions of CC- $0 \pi$ sample $(v$-mode) and CC-1-track sample ( $\bar{v}$-mode) in FGD1 after the ND280 fit, are shown in Fig. 2. The goodness of fit was found to be $8.6 \%$. For the parameters that ND280 can constrain, the fit reduces their effect on the uncertainty on the expected number of events at SK from $12-14 \%$ to 5-6\%.

\section{Far detector analysis}

The far detector SK is a 50-kt (22.5-kt fiducial mass) water Cherenkov detector, where the volume is divided into an outer detector (OD) with 1885 outward-facing 20-cm-diameter PMTs and an inner detector (ID) with 11129 inward-facing 50-cm-diameter PMTs. The events arriving at SK from the J-PARC beam spill are synchronized with a GPS within 150 ns precision. Candidate neutrino interactions must be fully contained in the fiducial volume. To enhance the "quasi-elastic" 

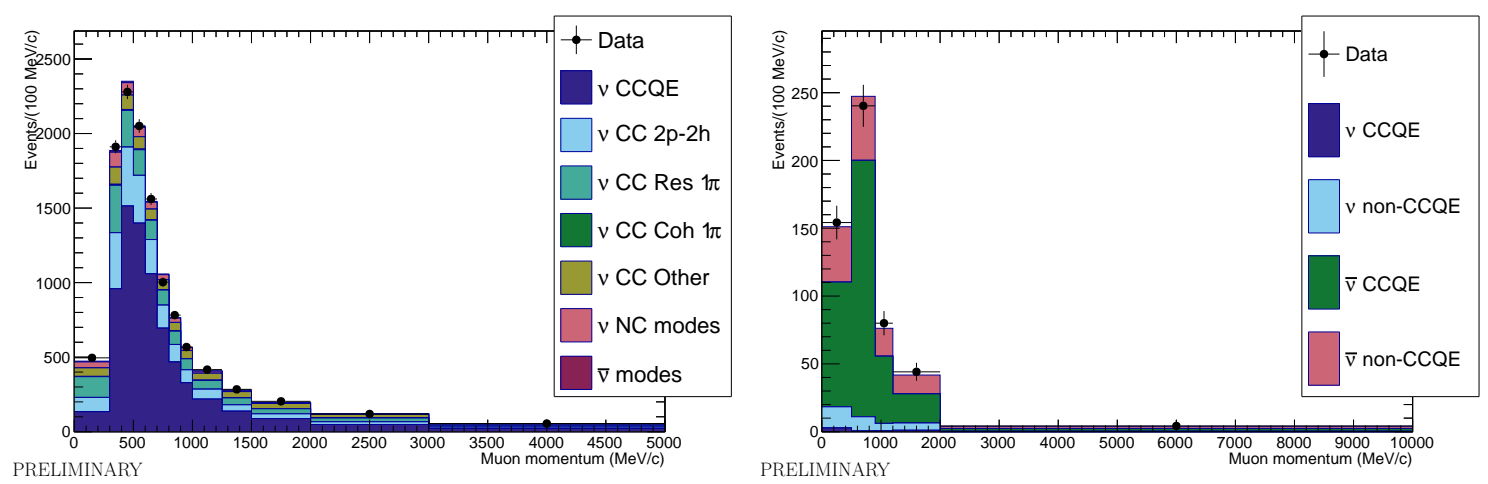

Figure 2: Predicted (histogram) and observed (data point) muon momentum distributions after the near detector fit for $v_{\mu}$ CC- $0 \pi$ events in $v$-mode (left) and $\bar{v}_{\mu}$ CC-1-track events in $\bar{v}$-mode (right).

purity of the samples, the selected events must have a single identified Cherenkov ring associated to the outgoing lepton. The $v_{\mu}$ and $\bar{v}_{\mu}$ samples are defined by the one ring muon-like candidates $(1 \mathrm{R} \mu)$, that implies a Cherenkov ring pattern consistent with a muon with a momentum of at least $200 \mathrm{MeV} / \mathrm{c}$, and at most one decay electron. The $v_{e}$ and $\bar{v}_{e}$ samples are defined by the one ring electron-like candidates $(1 \mathrm{R} e)$ that implies an electron-like ring with a visible energy greater than $100 \mathrm{MeV}$ with no decay electrons that may signal the presence of pions in the event. The neutrino energy $\left(E_{V}\right)$ is reconstructed from momentum and direction of the lepton (assuming a two body interaction) and must be lower than $1250 \mathrm{MeV}$. Moreover the $1 \mathrm{R} e$ events are passed through a further likelihood discriminator that separates out events containing a $\pi^{0}$. The resulting four events distributions are shown in Fig. 3.

\section{Oscillation results}

The results presented here are obtained with the whole dataset of $7.57 \times 10^{20}$ protons-ontarget (POT) collected in $v$-mode and $7.53 \times 10^{20}$ POT collected in $\bar{v}$-mode, for a total amount of $1.5 \times 10^{21}$ POT. A likelihood-ratio formed with the four samples collected at SK $(1 \mathrm{R} e / 1 \mathrm{R} \mu$ in $v / \bar{v}$-mode), is used as test statistic $(-2 \Delta \log \mathscr{L})$ where the nuisance parameters (flux, cross-section, detector and oscillation) are marginalised and the marginal likelihood is maximised as a function of the oscillation parameters of interest. Since the $v_{\mu}$ and $\bar{v}_{\mu}$ disappearance probabilities are identical at leading order $\sin ^{2} \theta_{23}$ (neglecting the small matter effects for T2K baseline), inconsistent measurements in the oscillation parameters between $v_{\mu}$ and $\bar{v}_{\mu}$ disappearance analyses could be an indication of CPT violation or non-standard neutrino interactions. 135 and 66 candidate $1 \mathrm{R} \mu$ events are observed in $v$-mode and $\bar{v}$-mode, respectively. We found a good agreement between between $v_{\mu}$ and $\bar{v}_{\mu}$ disappearance results in both hierarchy cases. Therefore, no hint of CPT violation or non-standard neutrino interactions is observed. The left plot on Fig. 4 shows the allowed regions in $\sin ^{2} \theta_{23}$ and $\Delta m_{32}^{2}$ assuming normal hierarchy, where the T2K data (black) is compared with other recent experimental results. The T2K fit obtains $\sin ^{2} \theta_{23}=0.532_{+0.046}^{-0.068}\left(0.534_{+0.043}^{-0.066}\right)$ and $\Delta m_{32}^{2}=2.545_{+0.081}^{-0.084}\left(2.510_{+0.081}^{-0.083}\right) \times 10^{-3} \mathrm{eV}^{2}$ with normal (inverted) mass hierarchy hypothesis. The T2K measurement continues to be consistent with maximal mixing and is currently the 

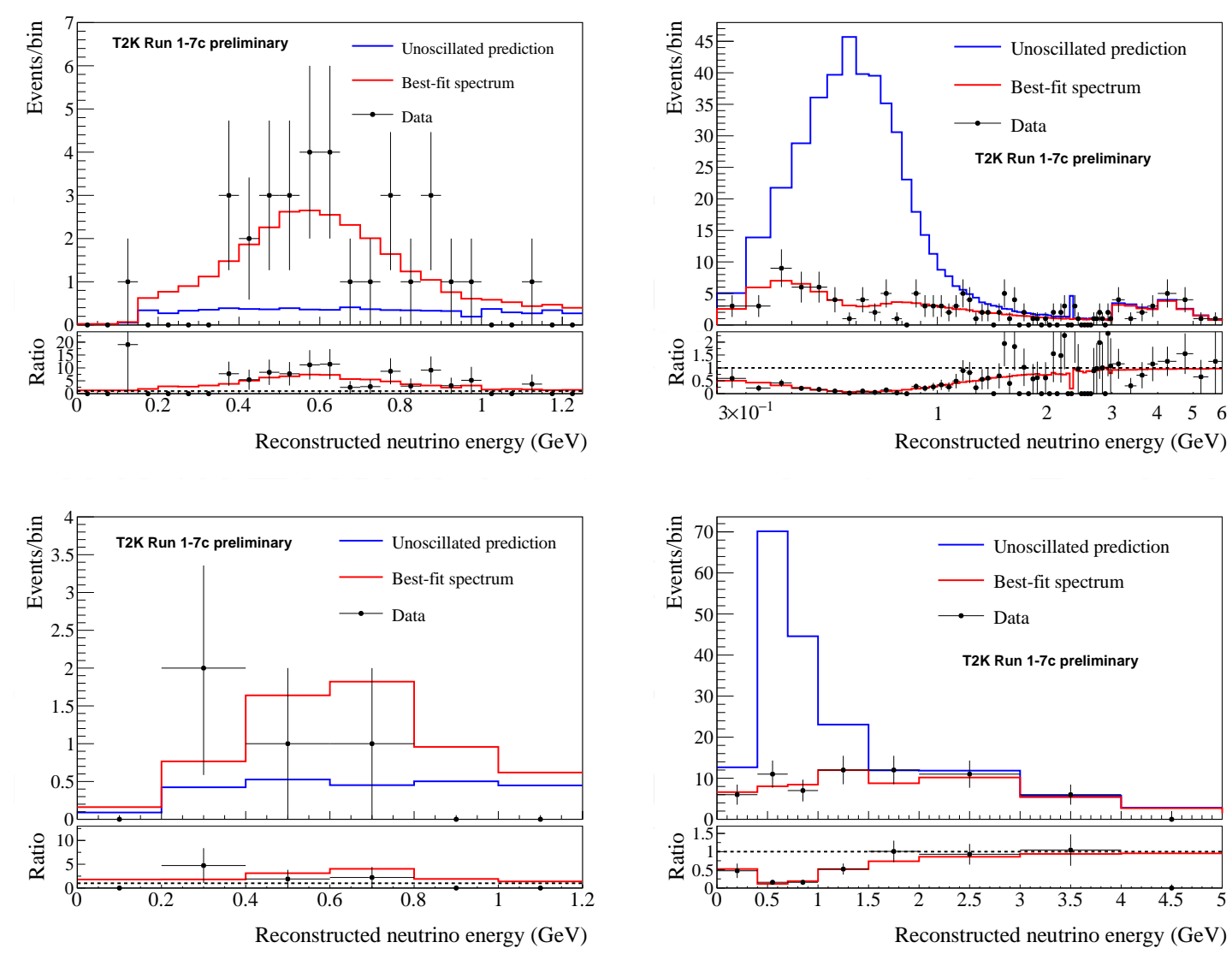

Figure 3: Top: reconstructed energy distributions for $v_{e}$ (left) and $v_{\mu}$ (right) candidates observed in $v$-mode. Bottom: corresponding distributions for $\bar{v}$-mode.

Table 1: Number of expected electron candidates in SK for the $v$-mode and $\bar{v}$-mode data for various values of $\delta_{C P}$ and the mass ordering, compared to the observation. The assumed value of other parameters are: $\sin ^{2} \theta_{13}=0.0217, \sin ^{2} \theta_{23}=0.528,\left|\Delta m_{32}^{2}\right|=2.509 \times 10^{-3} \mathrm{eV}^{2} / \mathrm{c}^{4}, \sin ^{2} \theta_{12}=0.846$ and $\left|\Delta m_{32}^{2}\right|=7.53 \times$ $10^{-5} \mathrm{eV}^{2} / \mathrm{c}^{4}$.

\begin{tabular}{cc|cccc|c}
\hline & & $\delta_{C P}=-\pi / 2$ & $\delta_{C P}=0$ & $\delta_{C P}=+\pi / 2$ & $\delta_{C P}= \pm \pi$ & Observed \\
\hline$v$-mode & $\mathrm{NH}$ & 28.7 & 24.2 & 19.6 & 24.1 & 32 \\
& $\mathrm{IH}$ & 25.4 & 21.3 & 17.1 & 21.3 & \\
\hline $\bar{v}$-mode & $\mathrm{NH}$ & 6.0 & 6.9 & 7.7 & 6.8 & 4 \\
& $\mathrm{IH}$ & 6.5 & 7.4 & 8.4 & 7.4 &
\end{tabular}

world-leading measurement of $\sin ^{2} \theta_{23}$.

The CP violating phase $\delta_{C P}$ is studied with the full joint fit analysis. As can be seen from Tab. 1 in $v$-mode, the number of observed 1 Re events are larger than the expectation for $v_{e}$ appearance candidate events, while in $\bar{v}$-mode the observed 1Re events are lower than the expected $\bar{v}_{e}$ appear- 
ance events. This behavior indicates that our data prefer the value of $\delta_{C P}$ inducing the largest $v-\bar{v}$ asymmetry: $-\pi / 2$. The $\mathrm{T} 2 \mathrm{~K}$ results are consistent with reactor ones, with the preference on the maximally $\mathrm{CP}$ violated region at $\delta_{C P}=-\pi / 2$. The right plot in Fig. 4 shows the $-2 \Delta \log \mathscr{L}$ as a function of $\delta_{C P}$ and mass hierarchy, with the reactor constraint on $\sin ^{2} 2 \theta_{13}[12,13,14]$. The $90 \%$ confidence levels are built with the Feldman-Cousins method [15], resulting in interval of $[-3.13,-0.39]$ in normal hierarchy and $[-2.09,-0.74]$ in inverted hierarchy. The T2K measurement rejects $\mathrm{CP}$ conservation in neutrino oscillations for both mass hierarchy hypotheses with $90 \%$ confidence level. From a toy Monte Carlo study we find that, if the true value of $\delta_{C P}=-\pi / 2$, then the probability for excluding $\delta_{C P}=0$ or $\pm \pi$, at $90 \%$ confidence level is $19.6 \%$ and $17.3 \%$ respectively.
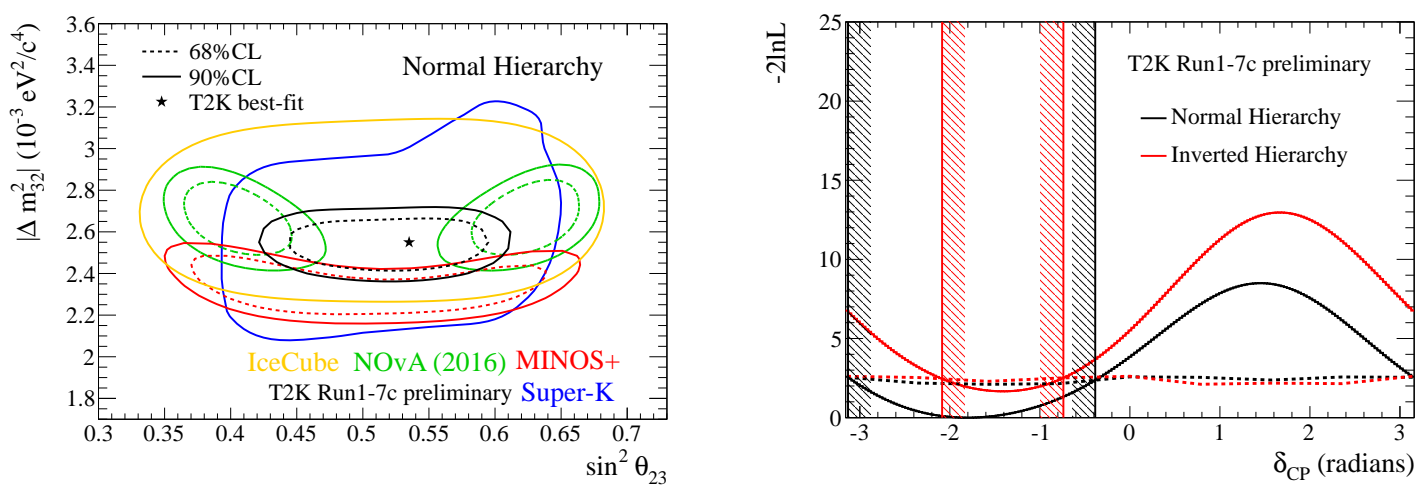

Figure 4: Left: Allowed regions in $\Delta m_{32}^{2}$ and $\sin ^{2} \theta_{23}$ from the T2K joint analysis (black) compared to other recent measurements. Right: $90 \%$ confidence level intervals in $\delta_{C P}$.

\section{Prospects}

The T2K experiment aims to reach the number of approved POT $\left(7.8 \times 10^{21}\right)$ around 2021 . However the current approved statistic is not enough to measure the $\mathrm{CP}$ violation with a sensitivity of $\sim 3 \sigma$ for the current favoured values of $\delta_{C P}$. For this reason, the T2K collaboration proposes a further extension of the T2K data taking up to 2026, called T2K-II, in order to collect $20 \times 10^{21}$ POT [16]. To achieve this goal, the accelerator and MR beam power need to be upgraded to reach 1.3 MW from the current beam power of $420 \mathrm{~kW}$. Moreover, various aspects of the T2K experiment need to be upgraded accordingly as well: upgrade of the magnetic horn current, from present 250 $\mathrm{kA}$ to $320 \mathrm{kA}$ to increase the effective POT by $\sim 50 \%$, near detector upgrade and some analysis improvements to increase the statistic and reduce the systematic uncertainties. Considering all these improvements, T2K-II can reach a $\sim 3 \sigma$ sensitivity for the current favoured values of $\delta_{C P}$ and measure $\theta_{23}$ with a precision of $1.7^{\circ}$ as well.

\section{Conclusions}

For the first time we present the results of a fully joint analysis across all four neutrino oscillation modes observed at $\mathrm{T} 2 \mathrm{~K}\left(v_{\mu} / \bar{v}_{\mu}\right.$ disappearance and $v_{e} / \bar{v}_{e}$ appearance), obtained with the full statistic collected so far $\left(1.5 \times 10^{21}\right.$ POT split equally in $v$-mode and $\bar{v}$-mode $)$. Our data prefer 
maximal mixing in $\theta_{23}$, while the relatively large $v_{\mu} \rightarrow v_{e}$ signal and small $\bar{v}_{\mu} \rightarrow \bar{v}_{e}$ signal with respect to the expectations, favour the scenario of $\delta_{C P}=-\pi / 2$ and normal hierarchy. Thanks to rapid increases in beam power and projected upgrades, beam power should continue to rise in the near future. The T2K Collaboration proposes further extension of the T2K run (T2K-II) up to 2026 in order to collect $20 \times 10^{21} \mathrm{POT}$, nearly three time the current approved $7.8 \times 10^{21} \mathrm{POT}$. Such extension of $\mathrm{T} 2 \mathrm{~K}$ is needed in order to measure the $\mathrm{CP}$ violating effects with $\sim 3 \sigma$ sensitivity, if the true value of $\delta_{C P}$ is $-\pi / 2$ and the mass hierarchy is normal, and to solve the octant of $\theta_{23}$ with a precision of $1.7^{\circ}$. In order to achieve these goals, upgrades of the beamline to support beam power up to $1.3 \mathrm{MW}$, improved detectors, and refined analyses are required. A first stage of beamline upgrade is foreseen in 2018.

\section{References}

[1] K. Abe et al. (T2K Collaboration), Nucl. Instrum. Methods A659, 106 (2011).

[2] K. Abe et al., Nucl. Instrum. Meth., A659, 106-135, 2011.

[3] N. Abgrall et al. (NA61/SHINE Collaboration), Phys. Rev. C 84, 034604 (2011).

[4] N. Abgrall et al. (NA61/SHINE Collaboration), Phys. Rev. C 85, 035210 (2012).

[5] N. Abgrall et al. (NA61/SHINE Collaboration), Eur. Phys. J. C76, 84 (2016).

[6] K. Abe et al. (T2K Collaboration), Nucl. Instrum. Methods A694, 211 (2012).

[7] T2K Collaboration (K. Abe (Tokyo U., ICRR) et al.), Phys.Rev. D87 (2013) no.1, 012001

[8] N. Abgrall et al. (T2K ND280 TPC Collaboration), Nucl. Instrum. Methods A637, 25 (2011).

[9] P. Amaudruz et al. (T2K ND280 FGD Collaboration), Nucl. Instrum. Methods A696, 1 (2012).

[10] K. Abe et al. (T2K Collaboration), Phys. Rev. D91, 072010 (2015).

[11] T2K Collaboration (Ko Abe (Kamioka Observ.) et al.), Phys. Rev. Lett. 116 (2016) no.18, 181801.

[12] F. P. An et al. Phys. Rev. Lett., 108, 171803, 2012.

[13] J. K. Ahn et al. Phys. Rev. Lett., 108, 191802, 2012.

[14] Y. Abe et al. Phys. Rev. Lett., 108, 131801, 2012.

[15] G. J. Feldman and R. D. Cousins, Phys. Rev. D 57, 3873 (1998).

[16] Ko Abe et al. e-Print: arXiv:1609.04111. 